\title{
RECENT ADVANCES ON STRUCTURAL BIOINFORMATICS, CELL MOTION SIMULATION, FUNCTIONAL MODULE IDENTIFICATION, COPY NUMBER VARIATION, AND PROTEASE SUBSTRATE PREDICTION AND SOME CRITICAL COMMENTS ON HMMER2
}

\section{Structural bioinformatics is an important branch of bioinformatics that focuses} on the analysis and prediction of the three-dimensional structure of proteins, RNA, and DNA. Research in structural bioinformatics includes topics such as structure determination, ${ }^{1,2}$ molecular folding, ${ }^{3}$ binding interactions, ${ }^{4,5}$ and structure/function relationships. ${ }^{6}$ The first four papers in this issue present new results on several challenges pertinent to structural bioinformatics.

In the first paper, Guo and $\mathrm{Rao}^{7}$ tackle the challenge of predicting protein folding rate from amino acid sequence. Their approach is based on a combination of neural network and genetic algorithm. Furthermore, in contrast to many past methods, they do not require any explicit structural information. Their method achieves $80 \%$ correlations between predicted and experimental folding rates, which is an improvement on many previous methods.

In the second paper, Alipanahi et al. ${ }^{8}$ consider the problem of backbone resonance assignment in NMR structure determination. Past methods have not worked well when the input contains too many noisy peaks. In this work, Alipanahi et al. have developed the IPASS system for error-tolerant backbone resonance assignment based on integer linear programming, as well as new approaches for grouping chemical shifts into spin systems and extracting connectivity information from spin systems. Their method delivers $82 \%$ precision and $63 \%$ recall. This performance is a significant improvement on previous methods such as MARS.

In the third paper, Hayat et al. ${ }^{9}$ introduce the BTMX method for predicting the exposure status of residues in transmembrane beta barrel proteins. Existing prediction methods - e.g. Ref. 10 - pertaining to transmembrane proteins generally do not provide the exposure status of individual residues. Hence, this paper addresses a novel problem. BTMX is a purely sequence-based two-stage SVM classifier cascade. It achieves an accuracy of $78 \%$ on independent test data. This work has also identified various physico-chemical properties that show statistically significant differences between the oligomeric beta strands and non-oligomeric strands.

In the fourth paper, Kedarisetti et $a l .^{11}$ present BETArPRED, which is a sequence-based predictor of beta-strand segments and residues. BETArPRED is 
based on a logistic regression technique and uses primary sequence, secondary structure information (produced by SSpro, PSIPRED, and SPINE), and residue depth information (produced by RDpred) as input features. The performance of BETArPRED in recognizing beta-strand segments and residues is better than most modern secondary structure predictors as well as a state-of-the-art automated structure predictor.

Besides the four structural bioinformatics papers above, this issue of the Journal of Bioinformatics and Computational Biology also includes three papers reporting new results on cell motion simulation, functional module identification, and copy number variation, as well as a review article on protease substrate prediction and a critical comments article on HMMER2. The details of these papers are summarized below.

Cell motion is central to many aspects of cell biology and to cancer metastasis. The development of an accurate model of cell motion has been an important goal of the mechanobiology community. I am pleased that, in this issue, Taylor et al. ${ }^{12}$ offer a mechanical model of cell motion that reproduces the adhesion and motility behaviors of cells in two-dimensional culture. Their simulations have also identified a threshold on the fragility of cell-cell cross-links that allows fluid-like motion while maintaining cohesiveness across the cell population.

There is much interest in analyzing gene expression data to uncover the causal factors of diseases ${ }^{13,14}$ and infer functional networks. ${ }^{15}$ In this issue, Prom-On et $a l .{ }^{16}$ describe an approach to improve the biological relevance of functional modules identified from gene expression data. Specifically, a backbone gene co-expression network (where the nodes are genes and edges are co-expression strength between genes) is first constructed based on minimum spanning tree and hub genes, and then this backbone network is divided into sub-networks that correspond to functional modules. Testing on a simulated dataset and on datasets from autism spectrum disorder and colorectal cancer shows that this method is very promising both in terms of enhancing the relevance of the functional modules and in terms of identifying more genes.

Determination and segmentation of DNA copy number variations is a more recently emerged problem in computational biology. ${ }^{17}$ This problem is made challenging by the noise in the array technologies for detecting DNA copy number and in the dilution of signals when samples contain mixtures of normal and disease cells. In this issue, Wang et al. ${ }^{18}$ propose using dynamic programming to find the optimal segment structure defined based on the minimal description length principle. Interestingly, the minimal description length is defined explicitly in terms of the proportion of disease/normal cells in the sample. Experiments suggest that this method is more accurate than earlier methods.

Proteases are recognized for their ability to catalytically hydrolyze protein substrates. Experimental identification of protease substrates is difficult. Hence, there has been interest in developing tools for the computational prediction of protease substrates. ${ }^{19}$ In this issue, Song et $a l .{ }^{20}$ provide an up-to-date review of recent 
advances in this field. The details, as well as the strengths and weaknesses, of current methods are discussed comprehensively.

Hidden Markov Models (HMM) are extremely useful for the analysis of data from biological sequences, and provide a probabilistic model of protein families. ${ }^{21}$ The HMMER package is probably one of the most widely used HMM suites in computational biology. The E-value produced by HMMER2 is computed as the lower of two values based on a logistic function and an extreme value distribution. It has been noticed over the years that E-values produced by HMMER2 are sometimes exaggerated, though the reason has remained unclear. In the final paper of this issue, Wong et al. ${ }^{22}$ close this issue via a critical analysis which identifies a critical region where the two values disagree - one giving E-value $<0.1$ and the other giving E-value $>0.1$ — for many domain models in hmmpfam.

\section{Limsoon Wong Managing Editor}

\section{References}

1. Poleksic A, Fienup M, Danzer JF, Debe DA, A different look at the quality of modeled three-dimensional protein structures, J Bioinform Comput Biol 6(2):335-345, 2008.

2. Zhao Y, Alipanahi B, Li SC, Li M, Protein secondary structure prediction using NMR chemical shift data, J Bioinform Comput Biol 8(5):867-884, 2010.

3. Glyakina AV, Galzitskaya OV, A comparative analysis of folding pathways of thermophilic and mesophilic proteins by Monte Carlo simulations, J Bioinform Comput Biol 8(3):395-411, 2010.

4. Aung Z, Tan SH, Ng SK, Tan KL, PPiClust: Efficient clustering of 3D protein-protein interaction interfaces, J Bioinform Comput Biol 6(3):415-433, 2008.

5. Ivan G, Szabadka Z, Grolmusz V, On the asymmetry of the residue compositions of the binding sites on protein surfaces, J Bioinform Comput Biol 7(6):931-938, 2009.

6. Parasuram R, Lee JS, Yin P, Somarowthu S, Ondrechen MJ, Functional classification of protein 3D structures from predicted local interaction sites, J Bioinform Comput Biol 8(Supp01):1-15, 2010.

7. Guo J, Rao N, Predicting protein folding rate from amino acid sequence, J Bioinform Comput Biol 9(1):1-13, 2011.

8. Alipanahi B, Gao X, Karakoc E, Li SC, Balbach F, Feng G, Donaldson L, Li M, Error tolerant NMR backbone resonance assignment and automated structure generation, $J$ Bioinform Comput Biol 9(1):15-41, 2011.

9. Hayat S, Walter P, Park Y, Helms V, Prediction of the exposure status of transmembrane beta barrel residues from protein sequence, J Bioinform Comput Biol 9(1):43$65,2011$.

10. Sadovskaya NS, Sutormin RA, Gelfand MS, Recognition of transmembrane segments in proteins: Review and consistency-based benchmarking of internet servers, J Bioinform Comput Biol 4(5):1033-1056, 2006.

11. Kedarisetti KD, Mizianty MJ, Dick S, Kurgan L, Improved sequence-based prediction of strand residues, J Bioinform Comput Biol 9(1):67-89, 2011.

12. Taylor WR, Katsimitsoulia Z, Poliakov A, Simulation of cell movement and interaction, J Bioinform Comput Biol 9(1):91-110, 2011. 
13. Sivachenko AY, Yuryev A, Daraselia N, Mazo I, Molecular networks in microarray analysis, J Bioinform Comput Biol 5(2b):429-456, 2007.

14. Dong G, Duan L, Tang C, Mining disease state converters for medical intervention of diseases, J Bioinform Comput Biol 8(1):77-97, 2010.

15. Bourque G, Sankoff D, Improving gene network inference by comparing expression time series across species, developmental stages or tissues, J Bioinform Comput Biol 2(4):765-783, 2004.

16. Prom-On S, Chanthaphan A, Chan JH, Meechai A, Enhancing biological relevance of a weighted gene co-expression network for functional module identification, J Bioinform Comput Biol 9(1):111-129, 2011.

17. Yin XL, Li J, Detecting copy number variations from array CGH data based on a conditional random field model, J Bioinform Comput Biol 8(2):295-314, 2010.

18. Wang S, Wang Y, Xie Y, Xiao G, A novel approach to DNA copy number data segmentation, J Bioinform Comput Biol 9(1):131-148, 2011.

19. Boyd SE, Pike RN, Rudy GB, Whisstock JC, de la Banda MG, POPS: A computational tool for modeling and predicting protease specificity, J Bioinform Comput Biol 3(3):551-585, 2005.

20. Song J, Tan H, Boyd SE, Shen H, Mahmood K, Webb GI, Akutsu T, Whisstock JC, Pike RN, Bioinformatic approaches for predicting substrates of proteases, J Bioinform Comput Biol 9(1):149-178, 2011.

21. Thorvaldsen S, A tutorial on Markov models based on Mendel's classical experiments, J Bioinform Comput Biol 3(6):1441-1460, 2005.

22. Wong WC, Maurer-Stroh S, Eisenhaber F, The Janus-faced E-values of HMMER2: Extreme value distribution or logistic function? J Bioinform Comput Biol 9(1):179206, 2011. 